\title{
The malignant progression effects of regorafenib in human colon cancer cells
}

\author{
Chisato Tomida', Kana Aibara', Naoko Yamagishi', Chiaki Yano', Hikaru Nagano³, Tomoki Abe', Ayako Ohno", \\ Katsuya Hirasaka ${ }^{2}$, Takeshi Nikawa ${ }^{1, \S}$, and Shigetada Teshima-Kondo ${ }^{1, \S}$ \\ ${ }^{1}$ Department of Physiological Nutrition, Institute of Biomedical Sciences, Tokushima University Graduate School, Tokushima 770-8503, Japan. \\ ${ }^{2}$ Graduate school of Fisheries Science and Environmental Studies, Nagasaki University, Nagasaki 852-8521, Japan. ${ }^{3}$ Faculty of Nutritional \\ Science, Sagami Women's University, Sagamihara 252-0383, Japan.
}

\begin{abstract}
A number of anti-angiogenic drugs targeting vascular endothelial growth factor receptors (VEGF-R) have developed and enabled significant advances in cancer therapy including colorectal cancer. However, acquired resistance to the drugs occurs, leading to disease progression, such as invasion and metastasis. How tumors become the resistance and promote their malignancy remains fully uncertain. One of possible mechanisms for the resistance and the progression may be the direct effect of VEGF-R inhibitors on tumor cells expressing VEGF-R. We investigated here the direct effect of a VEGF-R-targeting agent, regorafenib, which is the first small molecule inhibitor of VEGF-Rs for the treatment of patients with colorectal cancer, on phenotype changes in colon cancer HCT116 cells. Treatment of cells with regorafenib for only 2 days activated cell migration and invasion, while vehicle-treated control cells showed less activity. Intriguingly, chronic exposure to regorafenib for 90 days dramatically increased migration and invasion activities and induced a resistance to hypoxia-induced apoptosis. These results suggest that loss of VEGF signaling in cancer cells may induce the acquired resistance to VEGF/VEGF- $R$ targeting therapy by gaining two major malignant phenotypes, apoptosis resistance and activation of migration/invasion. J. Med. Invest. 62 : 195-198, August, 2015
\end{abstract}

Keywords : angiogenesis inhibitor, VEGF receptor tyrosine kinase inhibitor, regorafenib, colon cancer cells, malignant phenotype

\section{INTRODUCTION}

Colorectal cancer is the third leading cause of cancer mortality in Japan, and in 2013, about 48,000 deaths was attributed to this disease (1). Approximately $25 \%$ of colorectal cancer patients will have metastatic disease at the time of diagnosis, and an additional $50 \%$ of patients who initially present with early-stage disease will subsequently develop metastases over the course of their disease (2). Metastatic colorectal cancer remains a major public health problem, and diagnosis of metastatic disease is usually associated with poor prognosis.

Vascular endothelial growth factors (VEGFs) and their tyrosine kinase receptors (VEGF-Rs) are central regulators of tumor angiogenesis and lymphangiogenesis. Thus, VEGF/VEGF-Rs are crucial therapeutic targets in colorectal cancer therapy (3-5). Several angiogenesis inhibitors targeting VEGF (bevacizumab) and VEGFRs (regorafenib, sorafenib, sunitinib, vandetatnib, pazopanib and axiinib) have been developed and become an important option for management of a number of human malignancies.

Regorafenib is the first small molecule inhibitor of VEGF-Rs approved in 2014 in Japan for the treatment of patients with metastatic colorectal cancer who have progressed after standard therapies (6). In a pivotal randomized phase III study, a statistically significant prolongation in overall survival was observed in patients with colorectal cancer who received regorafenib therapy. These studies also demonstrated a significant improvement in progression-free survival. Despite the survival benefit of regorafenib for

Received for publication March 19, 2015 ; accepted March 31, 2015.

Address correspondence and reprint requests to Shigetada TeshimaKondo and Takeshi Nikawa, Department of Physiological Nutrition, Institute of Biomedical Sciences, Tokushima University Graduate School, 3-18-15 Kuramoto-cho, Tokushima 770-8503, Japan and Fax : +81-88633-7086. metastatic colorectal cancer patients, its overall clinical efficacy remains quite limited. Unfortunately, a significant number of patients do not respond to VEGF-R inhibitors. Furthermore, the vast majority of patients who initially respond to VEGF-R-targeting agents will develop resistance (7-9).

There is increasing evidence that VEGF/VEGF-R inhibitors act not only on vascular endothelial cells (anti-angiogenic function) but also on tumor cells that express functional VEGF-Rs (antiangiogenesis-independent function) (10-15). For instance, antiVEGF monoclonal antibody induces apoptosis resistance, spheroid formation and migration/invasion activity (13-15). VEGF-R inhibitors accelerate invasion activity in vitro and induces micro- and distant metastasis in vivo $(16,17)$. Thus, some of the effects observed with VEGF/VEGF-R targeting therapies could result from "direct" effects on tumor cells.

However, the direct effects of VEGF/VEGF-R inhibitors on tumor cells are not yet fully understood. We previously demonstrated that monoclonal anti-VEGF antibody (bevacizumab) induced a resistance to apoptosis of colon cancer cells (14). In this study, we examined the direct effects of VEGF-R inhibitor, regorafenib, on colon cancer cell phenotypes. We found that treatment of colon cancer HCT116 cells with regorafenib for only 2 days remarkably increased cell migration and invasion activities. Intriguingly, chronic exposure to regorafenib for 90 days dramatically increased cell migration and invasion activities. Furthermore, cells chronically treated with regorafenib acquired a resistance to hypoxia-induced apoptosis. These results suggest that loss of VEGF signaling in cancer cells may induce the acquired resistance to anti-angiogenic therapy by gaining two major malignant phenotypes, apoptosis resistance and migration/invasion. 


\section{MATERIALS AND METHODS}

\section{Cell culture and treatment}

Human colon cancer cell line (HCT116) was maintained in RPMI1640 medium with 5\% fetal bovine serum and antibiotics. To develop the HCT/rego-short cells, HCT116 cells were treated with regorafenib at $500 \mathrm{nM}$ (Selleckchem) for 2 days in RPMI1640 medium with $1 \%$ fetal bovine serum and antibiotics. For HCT/regolong cells, cells were chronically exposed to $500 \mathrm{nM}$ regorafenib for 90 days in RPMI1640 medium with 1\% fetal bovine serum and antibiotics.

For hypoxic culture conditions, cells were incubated at low confluence and $37^{\circ} \mathrm{C}$ in RPMI1640 medium supplemented with HEPES buffer (pH 7.4), 1\% fetal bovine serum and antibiotics using an AnaeroPack-Kenki 5\% anaerobic system in which $\mathrm{O}_{2}$ was $\sim 0.2 \%$ (Sugiyamagen, Japan).

\section{Assessment of apoptosis}

Apoptotic cells were assessed by imaging of activated caspase 3/7 using CellEvent caspase-3/7 green detection reagent (Molecular probes), according to the manufacturer's instruction.

\section{Cell migration and invasion assay}

Migration assay was conducted as described by Fan et al. (9) with minor modifications. Equal numbers (50,000 cells per well) of cells were suspended in $0.25 \mathrm{ml}$ of $1 \%$ RPMI1640-FBS without or with regorafenib and placed in the top compartment of a $8 \mu \mathrm{m}$ pore membrane chambers (BD Biosciences) ; $0.75 \mathrm{ml}$ of 10\% RPMI1640FBS was added to the bottom compartment. Following $24 \mathrm{~h}$ incubation under standard conditions $\left(37^{\circ} \mathrm{C} / 5 \% \mathrm{CO}_{2}\right)$, non-migrating cells were scraped from the top compartment, and cells that had migrated to the bottom compartment were fixed and stained using the Hemacolor Rapid staining of blood smear (Merck). Membranes were excised and mounted on a standard microscope slide. The numbers of migrated cells were determined from ten random highpower fields visualized at $\times 200$ magnification.

Invasion assays were done using a similar protocol with minor modifications. The inserts used in the invasion assays were coated with Matrigel (BD Biosciences) and prehydrated with $1 \%$ FBSsupplemented medium for 2 hours before the addition of the cell suspension. Following $48 \mathrm{~h}$ incubation under standard conditions $\left(37^{\circ} \mathrm{C} / 5 \% \mathrm{CO}_{2}\right)$, and the numbers of invading cells were quantified from ten random high-power fields visualized at $\times 200$ magnification.

\section{Statistical analysis}

Results are expressed as means \pm S.D. Statistical analyses of data were done using ANOVA and the Scheffé's test. P values $<0.05$ was considered significant.

\section{RESULTS}

Effect of short- and long-term exposure to regorafenib on hypoxiainduced apoptosis

We first examined the effect of short- (2 days) and long-term (90 days) exposure of colon cancer HCT116 cells to regorafenib on sensitivity to hypoxia-induced apoptosis, since one of the major in vivo effects of VEGF-R inhibitors is induction of hypoxic stress in tumors via suppression of angiogenesis $(18,19)$. HCT116 cells express all of VEGF-Rs (VEGF-R1 to -R3) and respond to VEGF-R inhibitor (15).

Cells were treated for 2 days with regorafenib (HCT/rego-short) or with vehicle (HCT/ctl), then they were exposed to hypoxic conditions $\left(\sim 0.2 \% \mathrm{O}_{2}\right)$ for additional $48 \mathrm{~h}$. Control HCT/ctl cells displayed a high degree of apoptosis (Fig. 1A). HCT/rego-short cells showed a similar degree of apoptosis (Fig. 1A). In contrast,
HCT116 cells chronically exposed to regorafenib for 90 days (HCT/ rego-long cells) exhibited a marked resistance to the apoptosis, compared with control HCT/ctl cells (Fig. 1B).

To explore how HCT/rego-long cells became a resistant to hypoxia-induced apoptosis, we tested a chemical HIF-1 $\alpha$ inhibitor (FM19G11) that effectively inhibited HIF- $1 \alpha$ transcriptional activity under hypoxic conditions in HCT116 cells $(14,15)$. Inhibition of HIF-1 $\alpha$ in HCT/ctl cells significantly increased hypoxia-induced apoptosis (Fig. 1B, FM19G11 +). However, blocking of HIF-1 $\alpha$ in $\mathrm{HCT} /$ rego-long cells did not significantly increase the apoptosis (Fig. 1B, FM19G11 +). This is consistent with our previous report that chronic treatment with foretinib, another type of a VEGF-R tyrosine kinase inhibitor, induced a resistance to hypoxia-induced apoptosis independently of HIF- $1 \alpha$ activity (15).

A
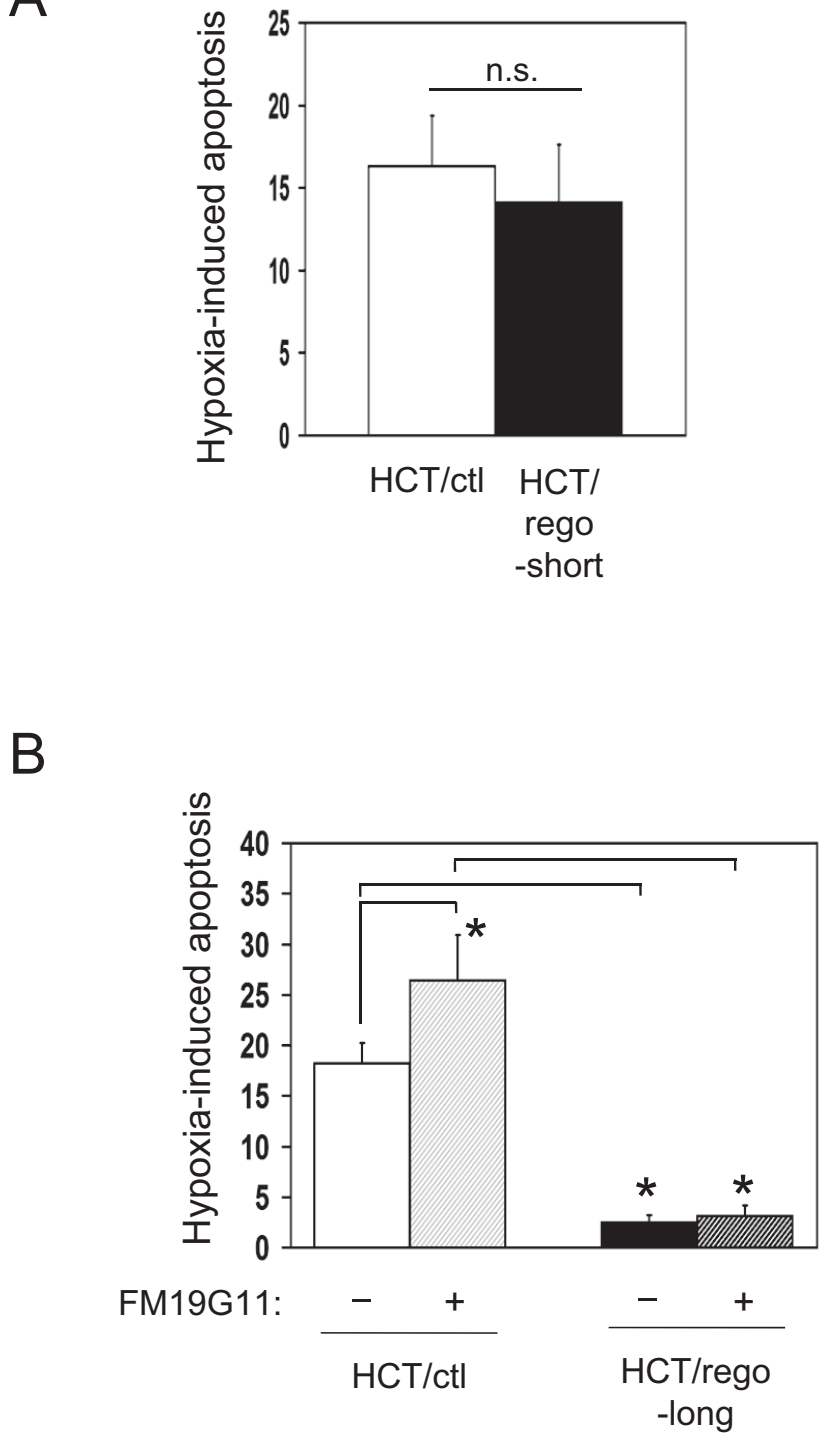

Figure 1. Effect of short- and long-term exposure to regorafenib on hypoxia-induced apoptosis

(A) Cells treated for $48 \mathrm{~h}$ with regorafenib (HCT/rego-short) or DMSO ( $\mathrm{HCT} / \mathrm{ctl})$ were exposed to hypoxia $\left(\sim 0.2 \% \mathrm{O}_{2}\right)$ for additional $48 \mathrm{~h}$, then apoptotic cells were determined by measuring of activated caspase $3 / 7$ ( $\mathrm{n}=4-5$, means $\pm \mathrm{SD}) .{ }^{*} P<0.01$., compared with control HCT/ctl cells. (B) Cells were exposed to hypoxia $\left(\sim 0.2 \% \mathrm{O}_{2}\right)$ for $48 \mathrm{~h}$ in the absence (-) or presence of HIF-1 $\alpha$ inhibitor FG19G11 (+), then apoptotic cells were determined $(\mathrm{n}=4$, means $\pm \mathrm{SD}) .{ }^{*} P<0.01$., compared with the respective control HCT/ctl cells in each group. 
A

Effect of short-term exposure to regorafenib on cancer cell migration and invasion activities

We then assessed the effects of short-term treatment with regorafenib on cell migration and invasion activities, since an increase in migration/invasion capacity is one of typical malignant phenotypes of cancer cells to escape from deterioration in the tumor environment (20). A cell migration activity was assessed using a modified Boyden chamber. Control HCT/ctl cells showed a slight migration activity (Fig. 2A, B). Intriguingly, treatment with regorafenib for only 2 days markedly increased the migration activity (a 5 -fold higher activity than control HCT/ctl cells) (Fig. 2A). We further examined the cell invasion activity using a Matrigel invasion assay. In agreement with activation of migration, invasive activity of HCT/rego-short cells was increased in 6-fold of HCT/ctl cells (Fig. 2C, D).
A

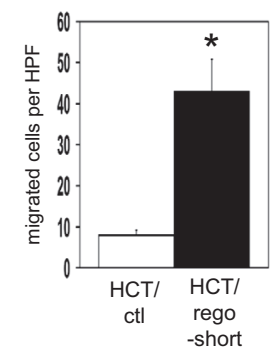

C

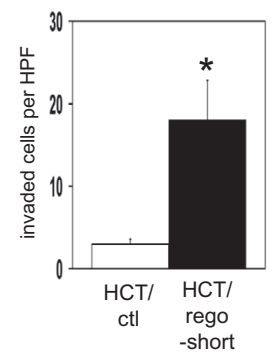

B

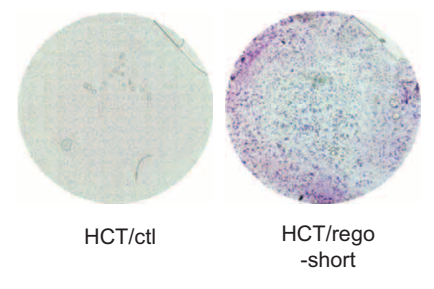

D

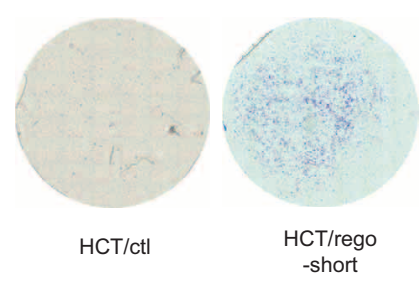

Figure 2. Effect of short-term exposure to regorafenib on cancer cell migration and invasion activities

(A) HCT/ctl and HCT/rego-short cells were used for transwell migration assay $(\mathrm{n}=3$, means $\pm \mathrm{SD}) .{ }^{\star} P<0.01$., compared with control HCT/ ctl cells. HPF, high power field.

(B) Representative microphotographs of migrated cells.

(C) HCT/ctl and HCT/rego-short cells were used for transwell invasion assay $(n=3$, means $\pm \mathrm{SD}) .{ }^{*} P<0.01$., compared with control HCT/ctl cells. HPF, high power field.

(D) Representative microphotographs of invaded cells.

Effect of long-term exposure to regorafenib on cancer cell $\mathrm{mi}$ gration and invasion activities

We further studied the effects of long-term treatment (for 90 days) with regorafenib on cell migration and invasion activities. $\mathrm{HCT} /$ rego-long cells exhibited a drastic increase in migration activity more than 20-fold of HCT/ctl cells (Fig. 3A, B). In consistent with the increased migration activity, HCT/rego-long cells showed a dramatic increase in their invasive activity, whereas HCT/ctl cells did not show the activity (Fig. 3C, D).

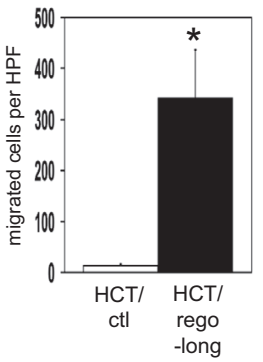

C

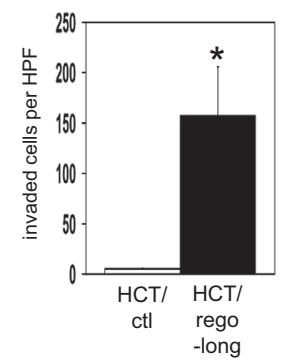

B

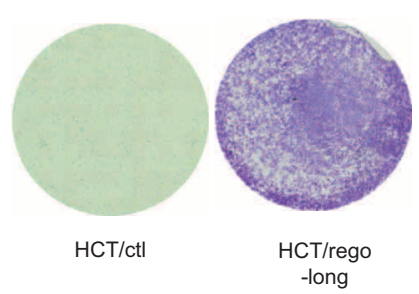

D

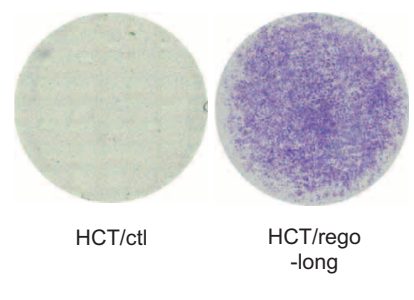

Figure 3. Effect of long-term exposure to regorafenib on cancer cell migration and invasion activities

(A) HCT/ctl and HCT/rego-long cells were used for transwell migration assay $(\mathrm{n}=4$, means $\pm \mathrm{SD}) .{ }^{*} P<0.01$., compared with control HCT/ ctl cells. HPF, high power field.

(B) Representative microphotographs of migrated cells.

(C) $\mathrm{HCT} / \mathrm{ctl}$ and $\mathrm{HCT} /$ rego-short cells were used for transwell invasion assay $(\mathrm{n}=4$, means $\pm \mathrm{SD}) .{ }^{\star} P<0.01$., compared with control HCT/ctl cells. HPF, high power field.

(D) Representative microphotographs of invaded cells.

\section{DISCUSSION}

This study focused on the short- (2 days) and long-term (90 days) effects of VEGF-R inhibitor regorafenib on cancer cells. We found that short-term inhibition of VEGF-R could increase migration and invasion activities. Furthermore, chronic blockade of VEGF-R induced more aggressive phenotypes (apoptotic resistance and migration/invasion phenotype).

$\mathrm{HCT}$ /rego-long cells acquired a resistance to hypoxia-induced apoptosis, and the resistance was independent of HIF-1 $\alpha$ (Fig. 1B). These results are consistent with our previous report showing that a chronic exposure to an another type of VEGF-R inhibitor, foretinib, also induced the apoptosis resistance that did not require HIF- $1 \alpha$ activation (15). By contrast, short-term treatment with regorafenib (Fig. 1A) and with foretinib (unpublished data) did not induce the resistance, suggesting that adaptation and/or selection of cancer cells that are resistant to hypoxia occur during the chronic, but not short-term, blockade of VEGF-R signaling.

Unexpectedly, treatment with regorafenib for only 2 days significantly activated cell migration and invasion (Fig. 2). Exposure to foretinib for 2 days did not significantly activate the migration (unpublished data), suggesting that regorafenib activates cellular adaptive signaling pathway(s) more strongly than foretinib. Further studies are needed to elucidate the precise molecular mechanism(s) activated by regorafenib.

Based on the present data and recent reports, it is possible that VEGF/VEGF-R targeting therapies directly inhibit VEGF signaling in tumor cells, which may remodel tumor cell survival signal(s). In fact, there is increasing evidence that VEGF itself suppresses 
migration and invasion of tumor cells in vitro (21), and conversely that inhibition of VEGF signaling accelerated migration and invasion in vitro and metastasis in vivo (21). Recently, two groups clearly elucidated how inhibition of VEGF signaling in tumor cells increases migration and invasion $(22,23)$. VEGF-R interacts with HGF receptor c-Met, a pro-invasive receptor, and inhibits c-Met activity by VEGF stimulation. Thus, blockade of VEGF/VEGF-R activates c-Met, leading to increased migration and invasion (22, 23). These and our findings suggest that over the long term inhibition of VEGF signaling, such remodeling results in adaptation to VEGF inhibition, and this adaptive response may represent one of potential mechanism of acquired resistance to VEGF/VEGF-R targeting therapies.

VEGF/VEGF-R initially held great promise as a therapeutic target in cancer. In fact, VEGF/VEGF-R-targeting therapy has been shown to be very effective in certain tumor types, such as renal cell carcinoma $(24,25)$. However, the overall benefit of blocking VEGF activity in other solid tumors is marginal and has led to some skepticism in the field. Therefore, molecular mechanism(s) activated by chronic loss of VEGF signaling in cancer cells will need to be elucidated to improve and further develop VEGF-targeting therapies.

\section{COMPETING INTERESTS-DISCLOSURE}

The authors declare no competing interests.

\section{ACKNOWLEDGEMENTS}

This work was supported in parts by grants from Grants-in-Aid for Scientific Research (23390198) from JSPS (to STK), Takeda Science Foundation (to STK), and The Mochida Memorial Foundation for Medical and Pharmaceutical Research (to STK).

\section{REFERENCES}

1. Saika K, Sobue T: Cancer statistics in the world. Gan To Kagaku Ryoho 40 : 2475-2480, 2013

2. Kohne $\mathrm{CH}$, Lenz $\mathrm{HJ}$ : Chemotherapy with targeted agents for the treatment of metastatic colorectal cancer. The oncologist $14: 478-488,2009$

3. Mitchell EP : Targeted therapy for metastatic colorectal cancer : role of aflibercept. Clin Colorectal Cancer 12 : 73-85, 2013

4. Fakih $\mathrm{M}$ : The evolving role of VEGF-targeted therapies in the treatment of metastatic colorectal cancer. Expert Rev Anticancer Ther $13: 427-438,2013$

5. Martinelli E, Troiani T, Morgillo F, Orditura M, De Vita F, Belli G, Ciardiello F : Emerging VEGF-receptor inhibitors for colorectal cancer. Expert Opin Emerg Drugs 18 : 25-37, 2013

6. Carter NJ : Regorafenib : a review of its use in previously treated patients with progressive metastatic colorectal cancer. Drugs Aging 31(1) : 67-78, 2014

7. Bergers G, Hanahan D : Modes of resistance to anti-angiogenic therapy. Nat Rev Cancer 8 : 592-603, 2008

8. Ellis LM, Hicklin DJ : Pathways mediating resistance to vascular endothelial growth factor-targeted therapy. Clin Cancer Res 14 : 6371-6375, 2008

9. Ebos JM, Lee CR, Kerbel RS : Tumor and host-mediated pathways of resistance and disease progression in response to antiangiogenic therapy. Clin Cancer Res 15 : 5020-5025, 2009

10. Fan F, Wey JS, McCarty MF, Belcheva A, Liu W, Bauer TW, Somcio RJ, Wu Y, Hooper A, Hicklin DJ, Ellis LM : Expression and function of vascular endothelial growth factor receptor 1 on human colorectal cancer cells. Oncogene $24: 2647-2653$, 2005

11. Bachelder RE, Crago A, Chung J, Wendt MA, Shaw LM, Robinson G, Mercurio AM : Vascular endothelial growth factor is an autocrine survival factor for neuropilin-expressing breast carcinoma cells. Cancer Res $61: 5736-5740,2001$

12. Frank NY, Schatton T, Kim S, Zhan Q, Wilson BJ, Ma J, Saab KR, Osherov V, Widlund HR, Gasser M, Waaga-Gasser AM, Kupper TS, Murphy GF, Frank MH : VEGFR-1 expressed by malignant melanoma-initiating cells is required for tumor growth. Cancer Res 71 : 1474-1485, 2011

13. Fan F, Samuel S, Gaur P, Lu J, Dallas NA, Xia L, Bose D, Ramachandran V, Ellis LM : Chronic exposure of colorectal cancer cells to anti-VEGF mAb promotes compensatory pathways that mediate tumor cell migration. British J Cancer 104 : 1270-1277, 2011

14. Yamagishi N, Teshima-Kondo S, Masuda K, Nishida K, Kuwano Y, Dang DT, Dang LH, Nikawa T, Rokutan K: Chronic inhibition of tumor cell-derived VEGF enhances the malignant phenotype of colorectal cancer cells. BMC Cancer $13: 229,2013$

15. Tomida C, Yamagishi N, Aibara K, Yano C, Uchida T, Abe T, Ohno A, Hirasaka K, Nikawa T, Teshima-Kondo S : Chronic exposure of VEGF inhibitors promotes the malignant phenotype of colorectal cancer cells. J Med Invest 62:75-79, 2015

16. Ebos JM, Lee CR, Cruz-Munoz W, Bjarnason GA, Christensen JG, Kerbel RS : Accelerated metastasis after short-term treatment with a potent inhibitor of tumor angiogenesis. Cancer Cell 15 : 232-239, 2009

17. Pàez-Ribes M, Allen E, Hudock J, Takeda T, Okuyama H, Viñals F, Inoue M, Bergers G, Hanahan D, Casanovas O : Antiangiogenic therapy elicits malignant progression of tumors to increased local invasion and distant metastasis. Cancer Cell $15: 220-231,2009$

18. Semenza GL: Targeting HIF-1 for cancer therapy. Nat Rev Cancer $3: 721-732,2003$

19. Semenza GL: Hypoxia-inducible factors in physiology and medicine. Cell $148: 399-408,2012$

20. Bottsford-Miller JN, Coleman RL, Sood AK : Resistance and escape from antiangiogenesis therapy : clinical implications and future strategies. J Clin Oncol $30: 4026$-4034, 2012

21. Lu KV, Chang JP, Parachoniak MM, Aghi MK, Meyronet D, Isachenko N, Fouse SD, Philips JJ, Cheresh DA, Park M, Bergers G : VEGF inhibits Tumor Cell Invasion and Mesenchymal Transition through a MET/VEGFR2 Complex. Cancer Cell $22: 21-35,2012$

22. Lu KV, Chang JP, Parachoniak CA, Pandika MM, Aghi MK, Meyronet D, Isachenko N, Fouse SD, Phillips JJ, Cheresh DA, Park M, Bergers G : VEGF inhibits tumor cell invasion and mesenchymal transition through a MET/VEGFR2 complex. Cancer Cell $22: 21-35,2012$

23. Sennino B, Ishiguro-Oonuma T, Wei $Y$, Naylor RM, Williamson CW, Bhagwandin V, Tabruyn SP, You WK, Chapman HA, Christensen JG, Aftab DT, McDonald DM : Suppression of tumor invasion and metastasis by concurrent inhibition of cMet and VEGF signaling in pancreatic neuroendocrine tumors. Cancer Discov 2 : 270-287, 2012

24. Vakkalanka BK, Rini BI : Targeted therapy in renal cell carcinoma. Curr Opin Urol $18: 481$-487, 2008

25. Escudier B, Cosaert J, Pisa P : Bevacizumab : direct anti-VEGF therapy in renal cell carcinoma. Expert Rev Anticancer Ther $8: 1545-1557,2008$ 